\title{
e-Migrinter
}

16 | 2017

Récits d'exilés

\section{Témoigner en migration, témoigner de sa migration}

Entretien avec Fabien Didier Yene

Fabien Didier Yene et Frédérik Detue

\section{OpenEdition}

\section{Journals}

\section{Édition électronique}

URL : https://journals.openedition.org/e-migrinter/930

DOI : $10.4000 /$ e-migrinter.930

ISSN : 1961-9685

\section{Éditeur}

UMR 7301 - Migrinter

Référence électronique

Fabien Didier Yene et Frédérik Detue, «Témoigner en migration, témoigner de sa migration », eMigrinter [En ligne], 16 | 2017, mis en ligne le, consulté le 20 mai 2021. URL : http://

journals.openedition.org/e-migrinter/930 ; DOI : https://doi.org/10.4000/e-migrinter.930

Ce document a été généré automatiquement le 20 mai 2021.

Tous droits réservés 


\section{Témoigner en migration, témoigner de sa migration}

Entretien avec Fabien Didier Yene

Fabien Didier Yene et Frédérik Detue

\section{NOTE DE L'ÉDITEUR}

Propos recueillis par Frédérik Detue.

FRÉDÉRIK DETUE : Vous avez écrit que votre témoignage est le fruit d'un travail mené « dans des conditions inimaginables » au Maroc, pourriez-vous revenir sur ces conditions?

Fabien Didier Yene: Il y a des choses qu'il faut comprendre avant que je ne commence d'exposer la situation vécue au Maroc.

Le voyage a d'abord été très périlleux, au Nigeria, au Niger, en Libye, en Algérie. J'ai vécu des agressions, du racket et encore du racket, j'ai connu la déportation. J'ai assisté à la mort de compagnons, j'ai vu des viols, des injustices, même parfois entre migrants, j'ai eu affaire à des organisations un peu mafieuses. Vous savez, quand les gens sont dans une situation d'exclusion, qu'ils sont réduits à l'instinct de survie, c'est la loi du plus fort qui l'emporte, je l'ai vécu.

Mais l'élément déclencheur qui m'a permis d'écrire mon témoignage, oui, c'est au Maroc qu'il s'est produit. Parce que je me suis retrouvé bloqué là pendant presque huit années. J'aurais dû le faire aussi en Europe si j'avais eu la chance de passer rapidement la frontière, mais je ne sais pas si j'y serais parvenu. J'aurais sans doute été comme la plupart qui arrivent là, qui ont des choses à dire mais qui ne font que courir pour survivre.

$\mathrm{Au}$ Maroc encore, l'écriture n'est pas venue tout de suite. Ce n'est pas que je ne le voulais pas. En fait, j'avais voulu tenir un journal depuis mon départ du Cameroun, et j'ai même essayé, je me souviens, au Tchad. Mais chaque fois que je me faisais contrôler, ce que j'écrivais était confisqué et détruit par la police. Puis j'ai compris 
aussi qu'il valait mieux que la police ne trouve rien sur moi, même pas un journal, parce que tu es plus en sécurité sans identité qu'avec une identité. Ce n'était pas possible non plus de tenir un journal pendant mes premières années au Maroc, quand je vivais dans les forêts qui jouxtent les enclaves de Ceuta et de Melilla. Quand je parle de la "chosification" des migrants, je pense en particulier à ces années-là. Nous devions nous terrer dans la journée à cause des rafles. Nous sortions la nuit pour trouver de la nourriture dans les poubelles ou dans l'espoir de passer l'une des barrières. C'était très dangereux, on pouvait être arrêtés, battus, refoulés. Nous vivions là dans de telles conditions que nous ne connaissions ni l'heure, ni le jour, ni la date. Notre place était réduite à l'inexistence et pourtant nous étions vivants.

L'élément déclencheur, je dirais que ça commence avec les tentatives massives de l'automne 2005, dont j'ai été l'un des organisateurs. Ces tentatives massives, elles se sont soldées par plusieurs morts, tués par balle. À ce jour, il n'y a toujours pas eu d'enquête indépendante, c'est révoltant. Le Maroc accuse l'Espagne, l'Espagne accuse le Maroc. Pourtant, si on se donnait un peu la peine, on pourrait très bien savoir qui avait les munitions, qui a tiré, d'où les balles sont parties. Au lieu de ça, c'est le moment que le Maroc a choisi pour se lancer dans des grandes opérations contre les migrants : les déportations dans le désert, les retours forcés dans les pays d'origine. Arrêté moi-même, détenu dans un camp militaire à Nador, j'ai échappé à l'expulsion un peu par miracle, grâce à un mensonge. J'ai été une fois de plus refoulé à la frontière algérienne. Mais là je n'ai pas voulu retourner dans les forêts, j'ai décidé de me rendre à Rabat.

Au départ, j'avais dans l'idée de me rapprocher de l'ambassade camerounaise, mais j'y ai été mal accueilli. En revanche, je me suis rendu à l'église et là, j'ai eu une chance énorme : j'ai rencontré le Père Jean-Pierre Michot, qui m'a gentiment orienté vers une ONG plus ou moins œcuménique, du nom de Caritas. Les bénévoles n'avaient pas de centre d'accueil mais ils m'ont orienté vers d'autres migrants pour que je trouve un hébergement et ils m'ont invité à revenir. C'est à partir de là, en commençant à fréquenter l'association jour après jour, que le projet m'est venu d'écrire mon témoignage.

FD : À la fin de votre témoignage, vous évoquez une jeune psychologue dont l'écoute a été précieuse pour vous, est-ce à ce moment-là que vous la rencontrez?

FDY : Oui, c'est ça. J'ai commencé à avoir des entretiens avec une bénévole de Caritas qui s'appelait Clotilde - pardon, je ne me souviens que de son prénom. Avec tout ce que j'avais vécu depuis des années, elle a senti qu'il fallait que je parle. En même temps, j'étais loin d'être le seul, elle recevait chaque jour beaucoup d'autres migrants qui avaient des histoires analogues à lui raconter. Elle me recevait une fois par semaine, une heure, une heure trente. J'avais tellement de choses à dire, j'ai commencé à ressentir le besoin d'une autre forme de communication. L'écriture, c'est du temps, pour réfléchir, penser à tout ce que tu as vécu, prendre de la distance. Je voulais que mon témoignage soit définitif, d'une certaine façon. Je me suis dit que c'était mon histoire et qu'il fallait que je l'écrive. Que je n'avais pas d'autre moyen de la faire entendre, de la faire comprendre. Je ne me prenais pas du tout pour un grand écrivain mais je voulais témoigner. Au moins, si c'était écrit, celui qui voudrait savoir ce que nous autres, Africains subsahariens, vivons en migration, pourrait connaître la vérité. Comment pourrait-il la connaître autrement? 
Ce qui s'est passé, c'est que j'ai fait une autre rencontre. L'intermédiaire a été un Français que j'avais connu en forêt, un dénommé Vincent Pollet. C'était un marginal. Il avait échoué à devenir prêtre mais il n'avait pas abandonné l'idée de prêcher l'Évangile néanmoins et il venait le faire auprès des migrants dans la forêt. Comme il était un peu suspect, nous avions fait une enquête sur lui pour vérifier qu'il n'était pas un indicateur de la police, mais il était vraiment sincère. À force de discuter religion, nous étions devenus amis et j'étais même devenu son protecteur. Je l'ai retrouvé à Rabat en retournant dans l'église du Père Jean-Pierre, et il m'a présenté à un vieux professeur français, plus exactement franco-allemand, qui s'appelle Marc Herbin-Bouthier.

Le vieux Marc était à la retraite après avoir ouvert et enseigné à la première faculté d'allemand à Rabat. On a commencé à discuter de ma situation, il n'en revenait pas. Il n'ignorait pas toute cette misère, pourtant, mais il ne savait pas comment agir. Je ne le juge pas ; simplement, il versait un don à Caritas à chaque fin de mois et, pour lui, c'était à Caritas de gérer la situation. Si ce n'est que, là, il se retrouve face à un gars qui vit les choses. Au début, il me donnait aussi un peu d'argent, de temps en temps. Le tournant, c'est quand je lui ai dit un jour : « Moi, j'ai le projet d'écrire tout ça. » Je me souviens de sa réaction : «Quand même, écrire!» Il était très enthousiaste. En fait, ça a touché le professeur : il n'attendait que ça.

D'abord, il m'a acheté une rame de papier mais, dans les conditions où je vivais, les feuilles, ce n'était pas commode. C'est quand il m'a acheté des cahiers que j'ai commencé à écrire, à la main. Ce témoignage, je l'avais commencé au Tchad quand je m'essayais à tenir un journal. Mais là il y avait un décalage de temps, c'était différent. J'ai ressenti le besoin de me dissocier, en tant qu'auteur, du Fabien qui souffre. C'est pourquoi j'ai décidé de faire un récit à la troisième personne, pour regarder la chose de l'extérieur.

Pendant ce temps, je continuais d'aller régulièrement à Caritas. J'ai annoncé à Clotilde que j'avais commencé à écrire. Elle m'a dit alors : «Moi je peux t'aider. Tu écris, moi je tape ton texte à l'ordinateur. » C'était un vrai soulagement, parce qu'à l'époque, la police pouvait survenir à tout moment et de nouveau détruire ce que j'écrivais. Désormais, il y aurait des traces, ce serait protégé. Et Clotilde m'a promis : «Je tape seulement, je n'ajoute rien. » Le vieux Marc - il n'avait pas loin de 80 ans, je crois - n'en revenait pas, car, quant à lui, c'était l'ancienne école, il n'était pas question d'ordinateur. Il m'a dit: «Maintenant, si tu veux, on peut s'occuper ensemble des corrections. »

Donc, j'écrivais, Clotilde tapait mon texte. Quand nous arrivions à dix, quinze pages, elle imprimait. Alors, je venais retrouver Marc pour les corrections. Mais ça donnait lieu à des discussions. Parce que Marc, sa référence, c'était un français académique. Or il y a des expressions qui ne sont peut-être pas académiques mais qui sont liées à l'expérience. J'ai souvent dit à Marc : « Non, il ne faut pas qu'on change ça, il faut que ça reste comme ça. »

FD : Est-ce que vous aviez déjà une idée d'un éditeur à qui vous pourriez vous adresser, dans ce moment?

FDY : Justement, ça a été tout un débat avec Marc. Il m'a posé la question : «Le livre, tu veux qu'il soit lu et connu où? » Pour moi, c'était clair depuis le début du projet: c'était plus important qu'on connaisse mon histoire en Afrique qu'en Europe. Le 
témoignage de tout ce que j'avais vu et vécu, j'avais pris conscience en le vivant que ça manquait. Ce qui m'intéressait, c'était de m'adresser à la jeunesse des quartiers dont je suis parti. Je me disais : "Il faut que tous ces jeunes comprennent ce qui se passe. Qu'ils sachent qu'on peut mourir pour du rêve. Qu'ils sachent comment ça arrive. " Je voulais leur faire comprendre qu'on est délaissés dans tous les sens. Parce que tu te dis : «Je suis en train de mourir chez moi, je vais aller ailleurs. » Mais même ailleurs, tu te fais massacrer et il n'y a personne. Tu n'as pas de valeur chez toi, c'est comme si tu étais mal né, alors tu pars. Mais là où tu veux aller non plus, tu n'as pas de valeur, tu n'existes pas : tu n'as pas le droit d'aller là-bas.

Marc comprenait, il me disait que, oui, mon récit pourrait être aussi connu plus tard en Afrique. Mais il pensait qu'il valait mieux l'éditer d'abord en Europe. Selon lui, c'était aussi important que les Européens soient au courant de ce que les migrants subissent au Maroc. Je voyais les choses autrement. Des gens qui racontent, je pensais que ça ne manquait pas en Europe : non seulement des migrants arrivaient là-bas, mais il y avait aussi les activistes des ONG qui pouvaient témoigner. Donc, je me disais que, si déjà tous ces gens disaient la vérité sur ce qui se passe et que malgré ça les Européens ne voulaient rien savoir, ce n'était pas moi, au Maroc, qu'on allait écouter. Je n'ai jamais changé de position à ce sujet. Néanmoins, les faits ont fini par donner raison à Marc.

Ce qui s'est passé, c'est que Marc et moi nous sommes rendus dans des foires aux livres en quête d'un éditeur. Or c'est très rare d'y rencontrer des éditeurs africains, en fait - ça n'a rien donné de ce côté, finalement. Pendant ce temps, nous avons échangé avec des éditeurs marocains, mais là, c'est une autre histoire. D'un côté, il y a tous ceux qui éditent de préférence en arabe. De l'autre, ceux qui éditent volontiers en français n'étaient pas prêts à éditer un témoignage comme le mien. Il faut replacer ça dans le contexte de l'époque, qui était extrêmement répressif. Je m'en étais rendu compte par ailleurs parce que j'avais commencé à témoigner dans des tables rondes et des conférences. Au Maroc, tout le monde avait peur. Quand un migrant témoignait pour la télévision, c'était toujours en tournant le dos à la caméra; il ne voulait surtout pas qu'on puisse l'identifier. Ainsi, j'ai été confronté au problème de la censure. Je me suis rendu compte que, si je voulais éditer au Maroc, c'était à la condition que le texte soit vraiment modifié. Les éditeurs me parlaient de choses qu'il fallait supprimer. Par exemple, je rapporte dans mon témoignage qu'on attend l'appel à la prière pour partir attaquer la barrière de Melilla. Dans ce passage ${ }^{1}$, j'ironise sur le fait que c'est une chance à saisir, étant donné que les policiers marocains sont alors trop occupés à rendre grâce à Dieu. Je me souviens que des éditeurs étaient choqués; ils trouvaient que je me moquais de leur religion. Il y a eu pas mal de choses dans ce sens. Surtout, ce qui ne passait pas, c'était que je dénonce le racisme des Marocains à l'égard des Noirs. Au Maroc, c'est un vrai tabou, le racisme. Mais je n'avais pas du tout l'intention d'embellir les choses, donc nous avons renoncé à cette piste.

Marc a écrit de jolies lettres à des éditeurs français, par exemple à Actes Sud. Mais ça n'a rien donné non plus. En fait, il a fallu encore une médiation. Il se trouve que Marc connaissait l'écrivain Abdellah Taïa, qu'il avait aidé à une époque en envoyant de l'argent à sa famille. Chaque fois qu'il publiait un nouveau livre, Abdellah l'envoyait à Marc. Marc a eu l'idée de lui parler de mon projet de publication, et c'est lui qui nous 
a mis en contact avec l'éditeur Séguier chez lequel il avait lui-même publié deux livres. Il m’a aidé comme ça alors qu'à l'époque, je ne l'avais jamais rencontré.

J'ai eu des désaccords avec Séguier. La couverture du livre, ce noir qui fait dissuasion, ça n'a pas été mon choix. Et ne parlons pas du prix du livre, 25 euros ! Je me rappelle que quand l'éditeur m'a annoncé combien le livre allait coûter, je ne comprenais même pas. C'était aberrant, qui allait acheter un livre à un prix pareil ? 25 euros, c'était la ration de toute une famille pendant un mois entier au Cameroun! Moi qui voulais qu'on puisse avoir accès facilement à mon témoignage... Mais Marc a insisté, il a dit qu'on ne devait pas passer à côté de cette chance de publication et qu'on devait accepter toutes les conditions. Déjà que je n'avais aucune garantie à l'époque de pouvoir venir en Europe, il ne fallait pas risquer en plus que mon témoignage reste comme ça sans être édité. Il m'a convaincu qu'il y aurait toujours la possibilité de faire les choses autrement après cette première publication.

Sur le moment, c'était tout de même contraignant. Dans un forum social, j'ai rencontré un militant allemand qui me trouvait très courageux de témoigner comme je le faisais. Quand je lui ai dit que j'écrivais, il a voulu que l'on m'édite en allemand. Je n'y étais bien sûr pas opposé, bien que je ne parle pas cette langue. Mais finalement l'éditeur allemand intéressé m'a contacté beaucoup plus tard, à un moment où j'avais déjà signé avec l'éditeur français, de sorte que le livre ne m'appartenait plus. Tout s'est donc passé d'éditeur à éditeur, avant même la parution en français. C'est pour ça que l'édition allemande ${ }^{2}$ est venue assez rapidement après l'édition française en 2010.

FD : Avant cette parution en 2010, le temps de l'écriture avait duré combien de temps? Pourriez-vous le borner avec précision?

FDY : Je ne mentionne pas ici la première phase du journal de migration qui n'a pas été sauvegardé. L'écriture du témoignage mené à publication a duré de 2006 à 2008. Mais il faut avoir conscience que rien ne garantissait alors que ce travail arriverait à terme. D'une part, mon but, c'était toujours prioritairement d'entrer en Europe, si bien que ça m'arrivait encore dans cette période de repartir en forêt pour faire des tentatives. D'autre part, Clotilde qui tapait mon texte n'était pas toujours là. Elle s'absentait parfois pour s'occuper de ses enfants, il arrivait aussi qu'elle parte en vacances en France : il y avait des périodes creuses. Elle était bien remplacée par une autre personne dans ces moments-là, mais tu as besoin de t'adresser à quelqu'un avec qui tu te sens en confiance pour un tel travail.

J'ai montré ma détermination à Clotilde, cependant. D'abord, elle voyait bien que je me donnais la peine de participer à des forums et à des tables rondes. Puis une chose a marqué les esprits à Caritas. Un jour, j'ai refusé un financement pour pouvoir me loger ; j'ai dit que je n'avais pas besoin de ça. J'ai exprimé en revanche le besoin de me former en informatique: d'apprendre à me servir d'un clavier et à naviguer sur Internet. Clotilde a compris que je voulais apprendre à taper mon texte moi-même et elle m'a soutenu. Je crois que c'est la première fois que Caritas a financé une telle formation à un migrant.

À partir de là, les choses ont changé. Un temps, Marc m'a donné de l'argent pour que j'aille faire des corrections dans un cybercafé. Puis, voyant que je me débrouillais bien, il a décidé de m'acheter un petit ordinateur. C'est sur celui-ci que j'ai fini l'écriture de mon témoignage. 
FD : Vous avez évoqué l'aide pratique que Clotilde, la bénévole de Caritas, vous a apportée. Mais qu'en est-il de l'aide psychologique qu'elle vous a également apportée : n'y a-t-il pas un lien avec l'écriture elle-même?

FDY : C'était complètement distinct. L'écoute de Clotilde m'a aidé, c'est certain. Ça libère, la parole. Après un entretien avec elle, je ne savais pas si ce que je lui avais confié était audible pour elle mais je ne m'en préoccupais pas : j'étais simplement soulagé d'avoir trouvé une oreille bienveillante. D'un autre côté, j'ai pu constater plus tard qu'elle m'avait bien lu. Quand des chercheurs européens venaient au Maroc, notamment dans les moments où la question migratoire était médiatisée, ils s'adressaient à Caritas. C'était normal qu'ils se tournent d'abord vers les institutions. Or Clotilde faisait tout ce qu'elle pouvait pour me mettre en contact avec ces chercheurs.

FD : Est-ce que vous leur parliez?

FDY : En fait, ça arrivait souvent que je ne veuille pas leur parler et que je les renvoie à Caritas en leur disant qu'ils obtiendraient là toutes les informations qu'ils voudraient. C'est une chose qu'on avait trop tendance à oublier: en fait, il était important de considérer les choses sous l'angle de la protection de la communauté. Car, pour les migrants, cela posait un problème de recevoir une personne dans la forêt, qu'il s'agisse d'un chercheur ou du membre d'une ONG. Cela représentait même un vrai danger. Nous avons pu le constater à plusieurs reprises, y compris quand les personnes étaient expérimentées. Par exemple, la militante des droits de l'homme Helena Maleno Garzón, dont je parle dans mon témoignage, me prévenait parfois qu'elle allait venir apporter son aide dans la forêt tel ou tel jour; elle apportait des petites choses, des médicaments, du pain - elle n'avait pas beaucoup de moyens. Or, systématiquement, la police venait tout saccager quelques jours plus tard. Nous avons fini par réfléchir collectivement à la stratégie qu'il convenait d'adopter, comme dans une guerre de position! Nous nous demandions comment recevoir ces personnes sans nous faire repérer par la police. Mais la priorité, c'étaient les personnes qui venaient pour nous aider.

FD: 2006, c'est aussi l'année où vous commencez vos activités militantes, en quoi consistaient-elles?

FDY : C'étaient des petites choses, parce que moi aussi j'avais peu de moyens. D'abord, entre nous, dans la communauté, on ne prenait pas assez le temps de s'écouter les uns les autres. J'ai pris exemple sur Clotilde, ça a bien marché. Les camarades, je leur ai consacré du temps jusqu'à ce qu'ils me racontent leur périple. Ça a commencé à se savoir : "Lui au moins, il nous écoute, même s'il n’a pas grandchose ".

Par ailleurs, il y avait le problème des disparitions. Des gens qui prenaient des zodiacs et qui mouraient sans qu'on n'ait d'informations sur eux. De ce point de vue aussi, le fait que je possède un ordinateur a été important. C'est grâce à ça que j'ai créé l'association ADESCAM, l'association de sensibilisation et de développement des Camerounais migrants au Maghreb (Maroc). Un enjeu était de recueillir ces informations qui manquaient; les migrants venaient s'identifier et me donner les coordonnées de membres de leurs familles. Bien sûr, ça ne se faisait pas toujours simplement, il fallait d'abord que je les rassure sur le fait que je n'étais pas de la police. En même temps, il pouvait leur arriver quelque chose sans que personne n'en sache jamais rien, ni la police, ni la famille. Moi, je leur promettais que de toute façon 
ça resterait entre nous et qu'en cas de malheur, je ferais de mon mieux s'ils me donnaient le contact de leur famille. Ma préoccupation, c'était juste qu'on ait des traces, que notre histoire ne s'efface pas. C'est à ce sujet que Laetitia Tura et Hélène Crouzillat sont venues me trouver, parce que c'est en rapport avec le projet du film Les Messagers. Elles aussi ont conservé des traces. Elles ont pris des images de tout ça, même si des choses ont été coupées au montage.

FD : Et vous avez conservé tout ça?

FDY : Oui, j'ai conservé tout ça. J'ai encore toute une série d'informations dans mes fichiers aujourd'hui. Mais à l'époque ça exigeait beaucoup de prudence, il fallait que je sois très technique, d'autant que l'association ADESCAM elle-même était clandestine - il ne pouvait en être autrement au Maroc dans cette période. Il aurait été facile de m'accuser d'appartenir à un réseau de trafiquants. En plus, l'information circulait de migrant à migrant. Je devenais une personne de référence, ça devenait vraiment dangereux pour moi. Je m'en suis rendu compte quand un jour un migrant m'a appelé à 2 heures du matin : il était arrivé à la gare de Rabat, il ne savait que faire, il me demandait de l'aide. Je lui ai demandé : «Qui t’a donné mon numéro ?» On le lui avait donné à Oujda. Même dans les réseaux de trafiquants, on avait maintenant mon numéro. Ça faisait désormais partie de la transaction : une fois que tu avais payé le passeur, il te sortait le numéro de Fabien qui aidait les gens. Là, je me suis dit que ce n'était pas possible. Je risquais vraiment de me retrouver en prison. Je suis sûr que les Marocains ont fait des enquêtes.

FD : Vous avez eu constamment le souci de rendre visible l'existence des personnes qui subissaient les mêmes conditions de blocage que vous au Maroc. Quel lien faites-vous entre votre activité militante et votre activité de témoin?

FDY : Je crois que l'activité de témoin prime sur l'activité de militant, parce que ça me transforme, c'est comme une rencontre. Migrant au pied du mur, on le regarde comme le témoignage de quelqu'un qui veut émigrer, mais à mon avis il faut entendre le mot « émigrer » dans tous les sens, y compris sociologique.

$\mathrm{Au}$ départ de ton expérience migratoire, tu es seul avec ton petit projet. Tu vis une première injustice, une deuxième injustice, tu ne penses encore rien qu'à toi. Si ce n'est que chaque fois que tu es victime de quelque chose, heureusement qu'une autre personne est là pour te venir en aide, pour t'encourager. Quand tu commences à échanger avec les autres, tu éprouves le besoin d'abord de parler de tes injustices à toi. Mais peu à peu, à force de partager les mêmes expériences avec d'autres, des rapports de confiance s'établissent. Tu commences à t'ouvrir aux autres, à les écouter, tu deviens psychologue. Je ne dis pas que c'est le cas de tout le monde, certains restent très centrés sur eux-mêmes. Mais, quant à moi, cette expérience d'écouter les autres a commencé bien avant d'arriver au Maroc. Je ne sais pas si c'est une force que j'ai, mais je suis très empathique. Quand les autres me confient leurs histoires, ça me bouleverse. Au Maroc, ça m'est arrivé de pleurer tout seul en repensant aux histoires que d'autres, que je connaissais à peine, m'avaient racontées.

J'ai ressenti le besoin de témoigner mais j'ai compris aussi que ce n'était pas moi seul qui devais témoigner de toute cette misère et qu'on devait pouvoir faire des croisements entre les différents témoignages. C'est pourquoi j'ai eu envie de raconter l'histoire d'« Alain », dans le chapitre 2 de mon témoignage. Il m'a semblé qu'il fallait 
lui donner de la visibilité, même si je n'ai pas donné le nom de la vraie personne : son histoire était trop riche!

Il y a un risque, si tu laisses ceux qui ne l'ont pas vécu écrire à ta place. Je m'en suis rendu compte au Maroc, où nous rencontrions beaucoup de monde, des bénévoles de Caritas, des chercheurs. Nous leur expliquions beaucoup de choses. Mais à un moment, tu remarques quand même que c'est nous qui vivons ces choses et que c'est eux qui les écrivent. Or, parfois, tu as passé du temps à parler avec des personnes et tu te rends compte qu'elles ont déformé ce que tu leur as raconté, qu'elles sont dans un système. Parfois même, elles commencent à te l'avouer; en fait, elles écrivent ce que les gens ont envie d'entendre. Le désir de témoigner, il vient de là, aussi : nous nous demandions comment nous pouvions écrire tout ça avec nos mots, comment nous pouvions contredire toutes ces idées fausses.

Cette attitude, c'est ce qui a déterminé aussi mon activité de militant. D'ailleurs, je l'ai écrit dans une lettre politique que j'ai rédigée avec l'aide du vieux Marc. Elle était adressée à l'évêque, à l'État marocain, à l'ambassade du Cameroun. La thèse de cette lettre, c'était que, si l'on voulait régler les problèmes liés aux flux migratoires, il fallait des gens comme moi qui suis clandestin. Qu'ils le veuillent ou non, de toute façon, je m'engageais à faire ce travail, mais ce serait encore mieux si on travaillait ensemble.

FD : Quand avez-vous trouvé le titre Migrant au pied du mur et quel sens lui donnez-vous ?

FDY : Ça c'est une histoire curieuse. Je cherchais évidemment un titre en lien avec mon histoire de migration, mais finalement l'idée de Migrant au pied du mur est venue d'une autre petite expérience, vers la fin de l'écriture. Je me souviens que j'avais une petite amie marocaine. Ce qui m'étonnait beaucoup, c'est que ses frères étaient policiers et qu'ils comprenaient la chose. Quand j'allais prier à l'église, elle restait toujours à l'extérieur. Un jour, j'ai parlé de cette relation avec le père Jean-Pierre. Je lui disais que nous ne pouvions pas vivre ensemble mais que je me plaisais avec elle. Or, là, il me répond sèchement que de toute façon elle est musulmane et moi chrétien et que nous ne pourrons jamais nous marier. Je suis rentré chez moi et j'ai réfléchi. Je me suis demandé ce qu'elle pourrait bien ressentir si je lui rapportais ces propos. Elle prenait beaucoup de risques en sortant avec un Noir, et je devais aller lui dire que Dieu, qui nous a pourtant accueillis si différents, veut qu'on ne se marie pas? Le titre Migrant au pied du mur m'est apparu à ce moment-là, en rapport avec cette histoire d'amour et avec ma foi.

\section{NOTES}

1. Fabien Didier Yene, Migrant au pied du mur, Biarritz-Paris, Atlantica-Séguier, 2010, p. 205.

2. F. D. Yene, Bis an die Grenzen. Chronik einer Migration, Klagenfurt, Drava, 2011. 
INDEX

Mots-clés : témoignage, histoire de vie, littérature

\section{AUTEURS}

\section{FABIEN DIDIER YENE}

auteur

\section{FRÉDÉRIK DETUE}

Maître de conférences en littérature générale et comparée, Université de Poitiers - FoRRel (EA 3816), équipe B3

frederik.detue@univ-poitiers.fr 